\title{
Distributed Event-Based Process Execution - Assessing Feasibility and Flexibility
}

\author{
Pieter Hens ${ }^{1}$, Monique Snoeck ${ }^{1}$, Manu De Backer ${ }^{1,2,3,4}$, and Geert Poels ${ }^{2}$ \\ 1 K.U.Leuven, Dept. of Decision Sciences and Information Management, \\ Naamsestraat 69, 3000 Leuven, Belgium \\ 2 Universiteit Gent, Dept. of Management Information and Operations Management, \\ Tweekerkenstraat 2, 9000 Gent, Belgium \\ 3 Universiteit Antwerpen, Dept. of Management Information Systems, \\ Prinsstraat 13, 2000 Antwerpen, Belgium \\ 4 Hogeschool Gent, Dept. of Management and Informatics, \\ Kortrijksesteenweg 14, 9000 Gent, Belgium
}

\begin{abstract}
Processes modeling and execution (with a process engine) are getting more and more incorporated in todays business environments. This movement puts a lot of stress on classical process engines which have to coordinate many process instances simultaneously. Performance degrades quickly as the number of process instances increases, and a single point of failure is introduced by using a central process execution engine. In this paper, we address these challenges by providing a non-intrusive approach to distribute a process flow and have the flow executed by multiple, smaller process engines. We pay special attention to flexibility of the eventual distributed execution, since process change is costly in a distributed environment. We demonstrate the feasibility of our approach by providing an implementation of the transformation and execution architecture, and demonstrate the lower cost of process change that is achieved when using a flexible process runtime architecture.
\end{abstract}

\section{Introduction}

Process-aware information systems (PAISs) are becoming more and more integrated in todays business environments [1. Companies are aware of the running processes in their organization, where they analyze, model and execute these processes. Together with Service Oriented Architectures, these processes can be executed automatically by a process engine. Executing a process logic means coordinating the described work, invoking the correct services, adding tasks to the inbox of task managers, and choosing the correct control flow paths [2].

In classical process execution architectures, one process engine is responsible for the execution of one (designed) process model. However, the higher use and incorporation of processes in todays businesses means that one process engine has to handle a significant amount of process instances simultaneously. This puts a high pressure on the process engine, and performance degrades quickly as the number of process instances increases 34. Alongside degradation of the

T. Halpin et al. (Eds.): BPMDS 2011 and EMMSAD 2011, LNBIP 81, pp. 133-147, 2011.

(C) Springer-Verlag Berlin Heidelberg 2011 
performance, centralized execution also adds a single point of failure to the process architecture. Services are distributed and decentralized, but the decision logic and coordination of the workflow is still located at one point. Failure of the coordinator means failure of the entire process, even if the services themselves are still available 45 .

In this paper we propose a flexible, distributed approach to process execution to overcome the drawbacks of centralized execution. Besides the application services, the process flow (process logic) itself is also distributed in the IT architecture. We look at a distributed approach that has the following features:

- A non-intrusive approach, where the process flow is distributed (split) automatically at deployment time, without interference by the original process modeler.

- The split processes are each executed by a dedicated process engine, which differs both physically and logically from the other split process engines.

- The process runtime architecture is robust and scalable. There is no performance bottleneck or single point of failure.

- Unlike other process distribution approaches, we also focus on a loosely coupled and flexible runtime architecture. The distributed process infrastructure should handle process change [6] (at modeling level, as well as at execution level) with minimal cost.

The paper is structured as follows. We first start with a small example, which is used to explain the concepts throughout the paper. Next, we explain the general idea as well as the advantages of our distributed process execution approach. In section 4, the algorithm to distribute the global process flow is explained, together with a demonstration on how the algorithm can be implemented using BPMN as the process modeling language. Section 5] shows a prototype execution architecture and we continue with an illustration of the possibilities and advantages that can be reaped by using a loosely coupled architecture (Sect. 6). In section 7 we situate our approach in other existing proposals for distributed process execution and end with a conclusion (Sect. 8).

\section{$2 \quad$ Running Example}

Figure1 1shows a (BPMN) process model for a pizza delivery company. It involves three parties, a chef who bakes the pizzas and creates, if required, side dishes, a cashier who receives the orders and arranges the payments, and a delivery boy who eventually delivers the pizzas. This simple process incorporates the most frequently used process constructs [7] (simple sequence flows, exclusive and parallel gateways (split and join), lanes and a start and end event), human tasks and a service task. Although the example is kept simple for explanatory purposes, the proposed approach is not limited to these simple examples. Also note that this example omits data-flow considerations. Since this research is focused on the control flow of process execution, we assume data can be transmitted along with the sequence flow [8]. For the organization of data-dependencies in a distributed process architecture we refer to $[9]$. 


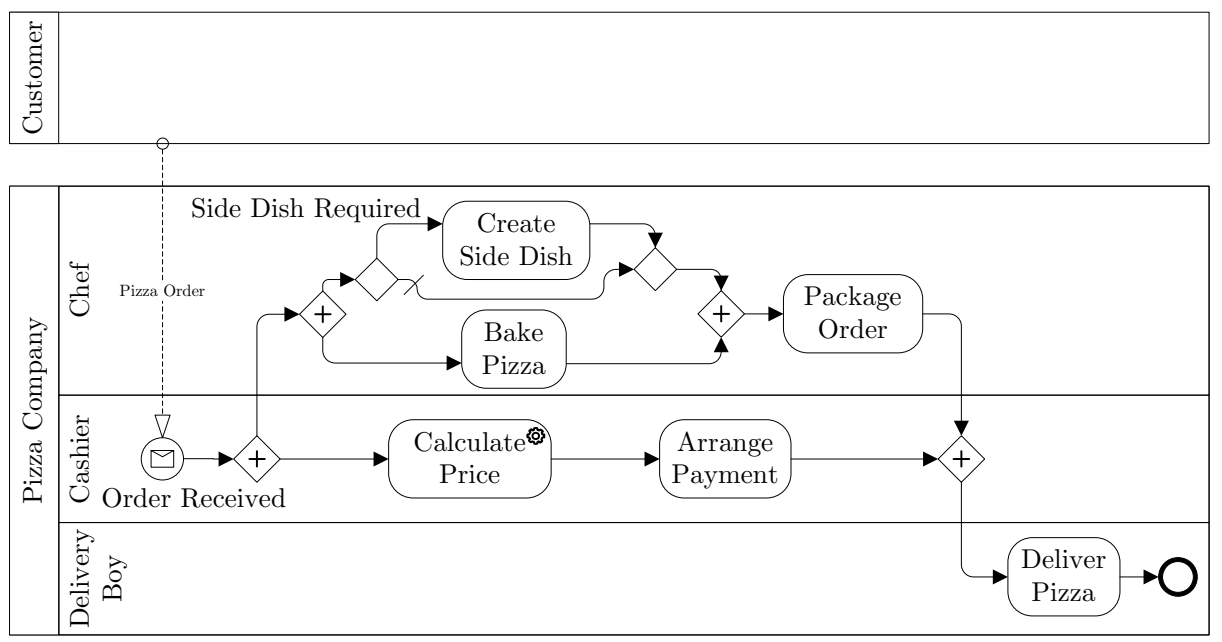

Fig. 1. Pizza Delivery Example

In the pizza company, a process engine is used to execute and control the process flow and a task manager is employed to handle the manual tasks. The process engine adds tasks to the task inbox of the respective performer and the task manager notifies the process engine of any completed tasks. On the other hand, to calculate the price, a (automated) service task is invoked by the process engine, and the service notifies the process engine of its completion (after which the process flow continues).

\section{Decentralized Event-Based Orchestration}

Figure 2a shows a part of the pizza company's process in the classical centralized approach to process execution. One engine coordinates the process flow (for each process instance) and invokes necessary services distributed in the IT architecture (PriceCalculator and TaskManagers). As mentioned in the introduction, centralized execution has many drawbacks which include a single point of failure, performance degradation and unnecessary network traffic [4]. To solve these drawbacks, several researchers have proposed solutions to distribute the process logic and use multiple process engines to execute, together, the entire process flow [45] (see Fig. 2b). These solutions solve the fundamental problems of central orchestration, but not to a full extent [10. The distributed (split) execution engines still remain tightly coupled in the process execution architecture. The start of one split engine relies on decisions (invocations) made by others (see the invocation links in Fig. 2 $\mathrm{b}$ ). As explained in [11] this request style of communication creates inflexible IT infrastructures and decreases scalability of the global process architecture. 


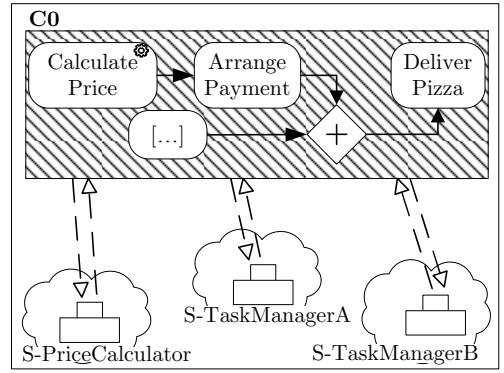

(a)

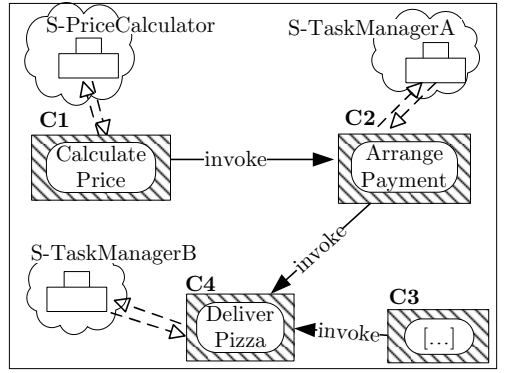

(b)

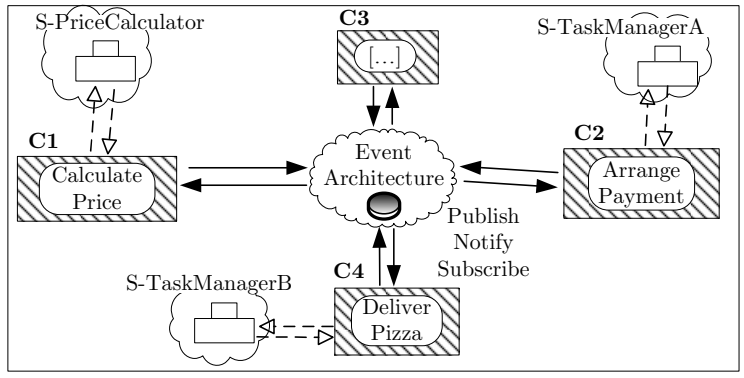

(c)

Fig. 2. Centralized, Decentralized and Event-Based Orchestration

To solve the tight coupling, we propose an extension to the distributed process approach, which uses an event-based architecture as the communication paradigm in the distributed process execution 10 (see Fig. 2k). Event based communication is a communication style that uses a publish/subscribe interaction scheme. An event is something that happens, and when an event occurs, a notification of this event occurrence is published in the architecture, where interested parties can receive this event notification. In contrary to a request-based communication style, an event message is non-directed and no expectancies (or SLAs) exist between the sender and the possible receiver of an event message [1]. In request-based communication, the responsibility for the next step is located at the caller (the process engine of CalculatePrice has the responsibility of invoking the start of ArrangePayment), while in event-based communication, this responsibility is located at the callee (the process engine of ArrangePayment is itself responsible for starting its execution at the correct time).

Decoupling of interaction partners is the main advantage of using event communication. This decoupling is defined as space decoupling (unawareness of interaction partners), time decoupling (interaction partners don't need to be active at the same time) and synchronization decoupling (asynchronous send and receive) [12. Together with the switch of responsibility from the caller to the callee, using an event based architecture creates a highly flexible and scalable process execution infrastructure. New pieces of the global process flow, can simply be 
added to the process architecture without making any changes to the already running infrastructure (they hold their own starting logic). Note that the supporting entities in an event based architecture (the cloud in Fig. 25) are also distributed and don't add another single point of failure. Many solutions exist that distribute the event based architecture itself [11.

To reap the benefits of event based communication, we need to transform the global process flow to a distributed event-based process. The most important part in this transformation is finding the starting rule of each split process. This is described in the following sections.

\section{Transformation}

To transform a global process into distributed segments, we choose a task as the unit of decomposition (the task can also be an embedded subprocess). Each

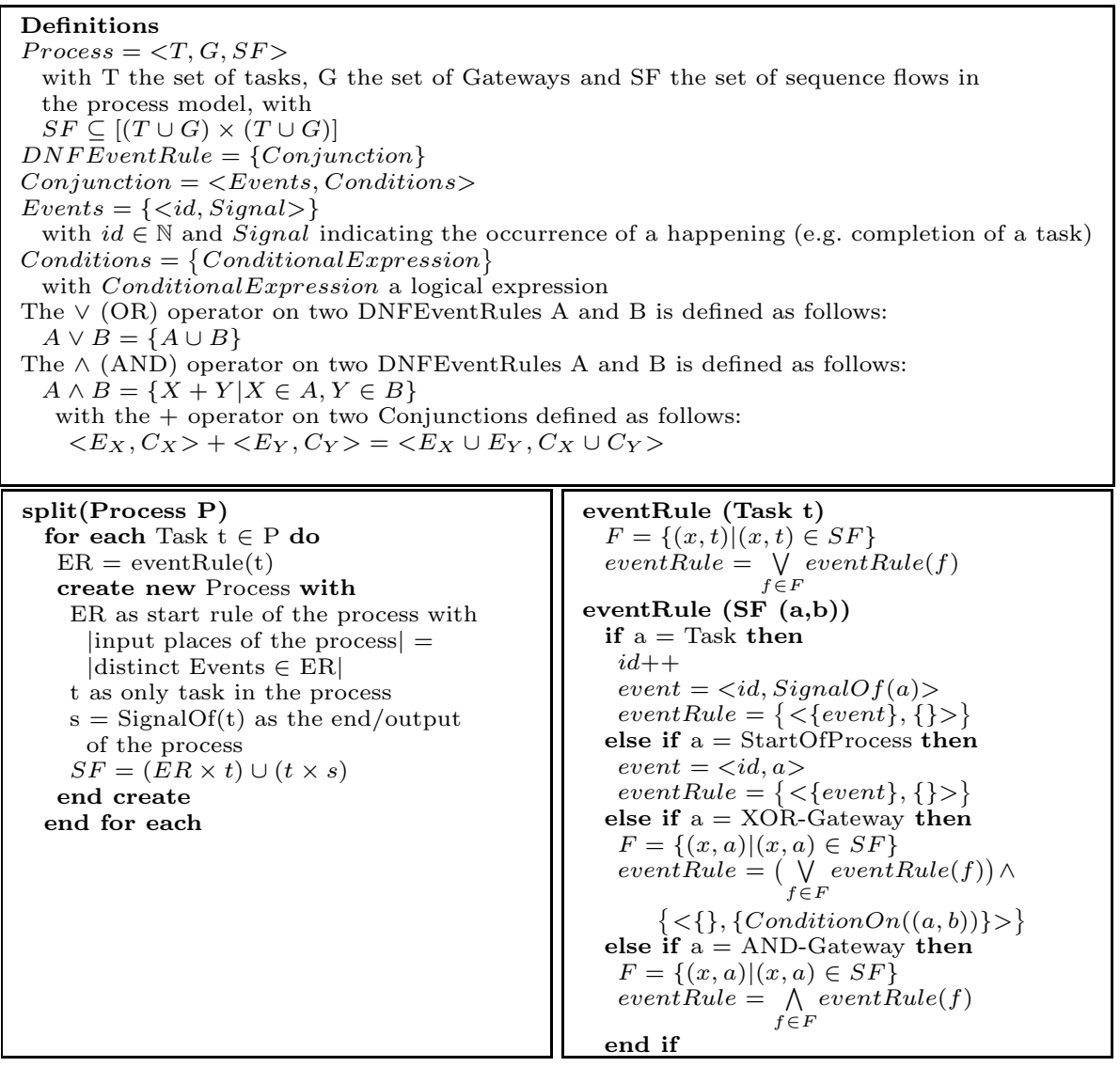

Fig. 3. Transformation algorithm 
task in the global process flow will become a small process itself, with a dedicated process engine. Choosing a task as the unit of decomposition, guarantees a fine grained distribution of the global process flow. The transformation and process executions shown in this paper can easily be extended to allow for other decomposition units, e.g. splitting according to user-defined regions, splitting according to workflow variants 13 or splitting according to the domain a task belongs to [14. In fact, it suffices to translate a process region to an embedded subprocess to define it as a non-splittable unit of decomposition.

Figure 3 shows the algorithm to split a global process into multiple processes (in $O\left(n^{2}\right)$ time). Each resulting process consists of a starting rule, a task to execute and an (end) event to publish the completion of the task. A starting rule for a split process consists of an event part (the event rule) and a user-defined conditions part (originating from XOR-splits in the global process). Finding the event rule for a split process equals finding, for a specific task, which preceding tasks in the process flow need to be completed before it can start its own execution. The algorithm finds these completion events in a depth-first search in the upward flow in the global process model. The event rule is transcribed as a logical expression in Disjunctive Normal Form, where an element in the expression is a happening in the information system (which we call events). For example, PackageOrderComplete AND ArrangePaymentComplete indicates a rule saying that the split process can start when tasks PackageOrder and ArrangePayment are completed (notifications are caught indicating the completion of these tasks). In the algorithm in Fig. 3 an event is notated as a tuple <id, signal $>$ with signal the event we want to receive (e.g. completion of task PackageOrder) and $i d$ a unique identifier. The id is necessary to make a distinction between two event rules, which have the same logical combination of event types, but have different execution semantics. For example, the distinction between the event rule $((<1, \mathrm{~A}\rangle$ AND $<1, \mathrm{~B}\rangle)$ OR $(<1, \mathrm{~B}\rangle$ AND $<1, \mathrm{C}\rangle))$ for a split process and the rule $((<1, A>$ AND $<1, B>)$ OR $(<2, B>$ AND $<1, C>))$ for another split process is shown in Fig. 4. In the latter event rule (Fig. 4b), two input places are enabled by an event B, which enables the possibility of two runs of the task $\mathrm{X}$ in the split process. With the first rule (Fig. 4a), only one input place is enabled by an event B. The distinction between these two rules is required for the transformation of non-safe process models, where it is possible to have multiple instances of one task, within one process instance.

The second part of a starting rule for a split process consists of user defined conditions originating from XOR-splits. These conditions are in conjunction with the event rule. Only when an event rule evaluates to true AND the respective conditions evaluate to true, then is the task in the split process able to execute. When searching for the completion events for the event rule, any condition encountered on an XOR-gateway is also stored in the starting rule (see Fig. 3).

How a starting rule is transcribed in the resulting split process is dependent on the process language used to describe the split processes. The algorithm in Fig. 3 is kept general, and only gives guidelines on how to build the split processes. Below we give a concrete example of the transformation and the transcription 


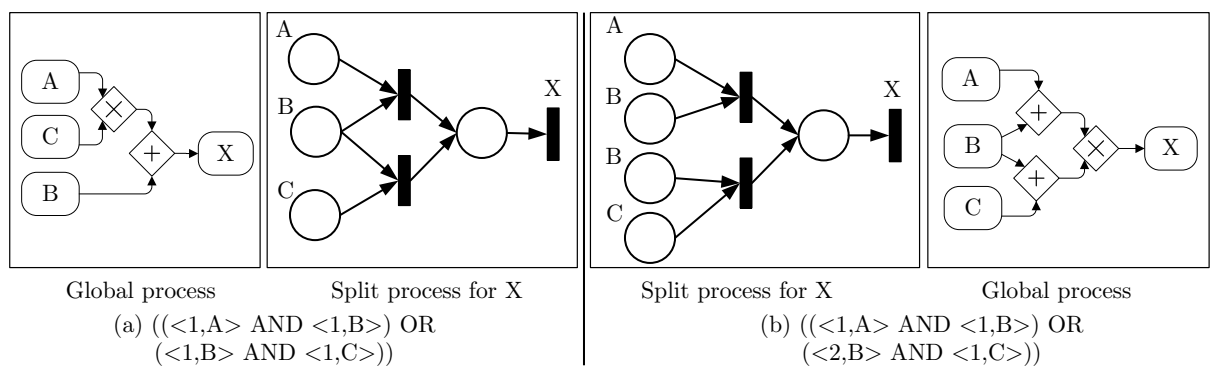

Fig. 4. Use of the identifier element in an event rule

of the starting rule, where we use BPMN2.0 as the language in which the global process as well as the split processes are described.

Algorithmic implementation with BPMN. We implemented the algorithm given in the previous section in the Atlas Transformation Language (ATL) [15]. ATL is a declarative language to describe a transformation of a source model (supported by a meta-model) to a target model. An eclipse plugin is available to create and execute these transformations. We have chosen to make an implementation transforming a BPMN2.0 [8] model to another BPMN2.0 model, where the first model represents the global process and the second the split processes. An advantage of describing the split models in the same language as the global model, is that existing process engines supporting the global model can also execute the distributed process flow, as long as they support communication of events with the publish/subscribe architecture.

As a starting point, we used the ecore meta-model of BPMN2.0, available at [16. Any BPMN model conforming to this meta-model can be used as input for the ATL-transformation (a BPMN Diagram Interchange XML-file 8]). To create the split processes, the ATL-transformation follows the algorithm described in the previous section, where a split process in BPMN is transcribed with the following properties:

- Signal events are used as start and end event for the split process. The semantics of a signal event in BPMN conform to the semantics of a notification in an event-based architecture. A throw signal is broadcasted without being directed to one particular process and can hence be caught by any and multiple receiving processes.

- A conjunction in the event rule is represented by multiple event definitions within one start event, with the parallel multiple marker for that start event set to true (see Fig. 5 for an excerpt of the event BPMN metamodel).

- A disjunction in the event rule is represented by using multiple start events.

- Conditions are placed on the respective sequence flow from the start event to the task.

Figure 6] shows an example of the BPMN representation of the split process for the Package Order task. It has the starting rule ((Pizza Baked AND Side 


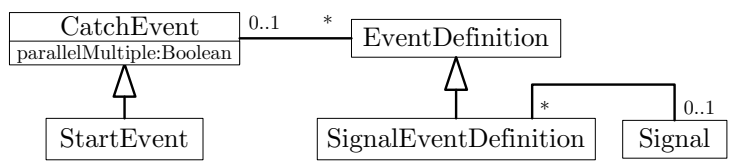

Fig. 5. An exert of the event meta model of BPMN2.0

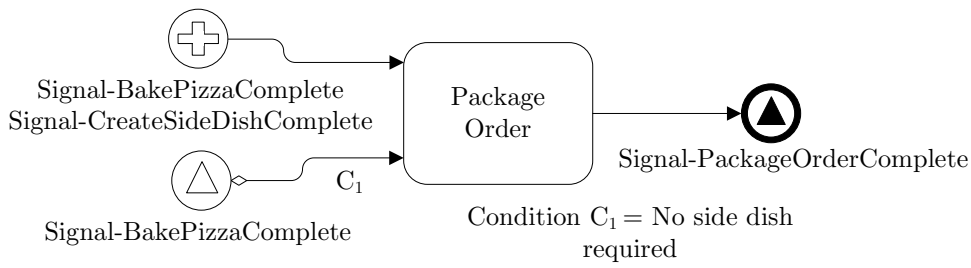

Fig. 6. Example of a resulting split process in BPMN

Dish Created) OR (Pizza Baked, with condition: No side dish required)). Note that the specifications of BPMN2.0 state that a conditional flow (a sequence flow carrying a condition) can't be connected from a start event to a task. We still use this notation as syntactic sugar. To make the model compliant with the BPMN specifications, the model can be easily changed by adding an XOR gateway in between the start event and the task.

The new split processes are stored in an XML-file conforming to the original BPMN2.0 metamodel. Any process engine, or BPMN editor, which supports the BPMN2.0 Interchange format can then be used to open, execute or visualize the resulting file describing the split processes. Figure 7 shows an example of an XML-input file and its resulting transformation, converted with our ATL implementation. In the output file, you'll find for each task a new process description, together with process-wide signal events indicating the completion of each task.

\section{$5 \quad$ Architecture and Process Execution}

After the transformation, each split process can be deployed to a dedicated process engine (see Fig. 8). Communication between the process engines happens with a publish/subscribe mechanism. For our prototype execution architecture, we've chosen the Siena wide area event notification service [17. Siena is a publish/subscribe implementation specifically directed to event subscription and notification in a wide area network, and provides all the necessary routing topology to transmit an event notification from the publisher to the subscriber. By being able to use multiple event services on the wide area network and because of the efficient routing, the single point of failure and performance bottleneck of the central orchestration are solved. Of course, to accomplish the event-based communication, any event architecture can be used, like WS-Notification [18, EVE [19] or the more recently proposed BPEL and WSDL extensions for an 


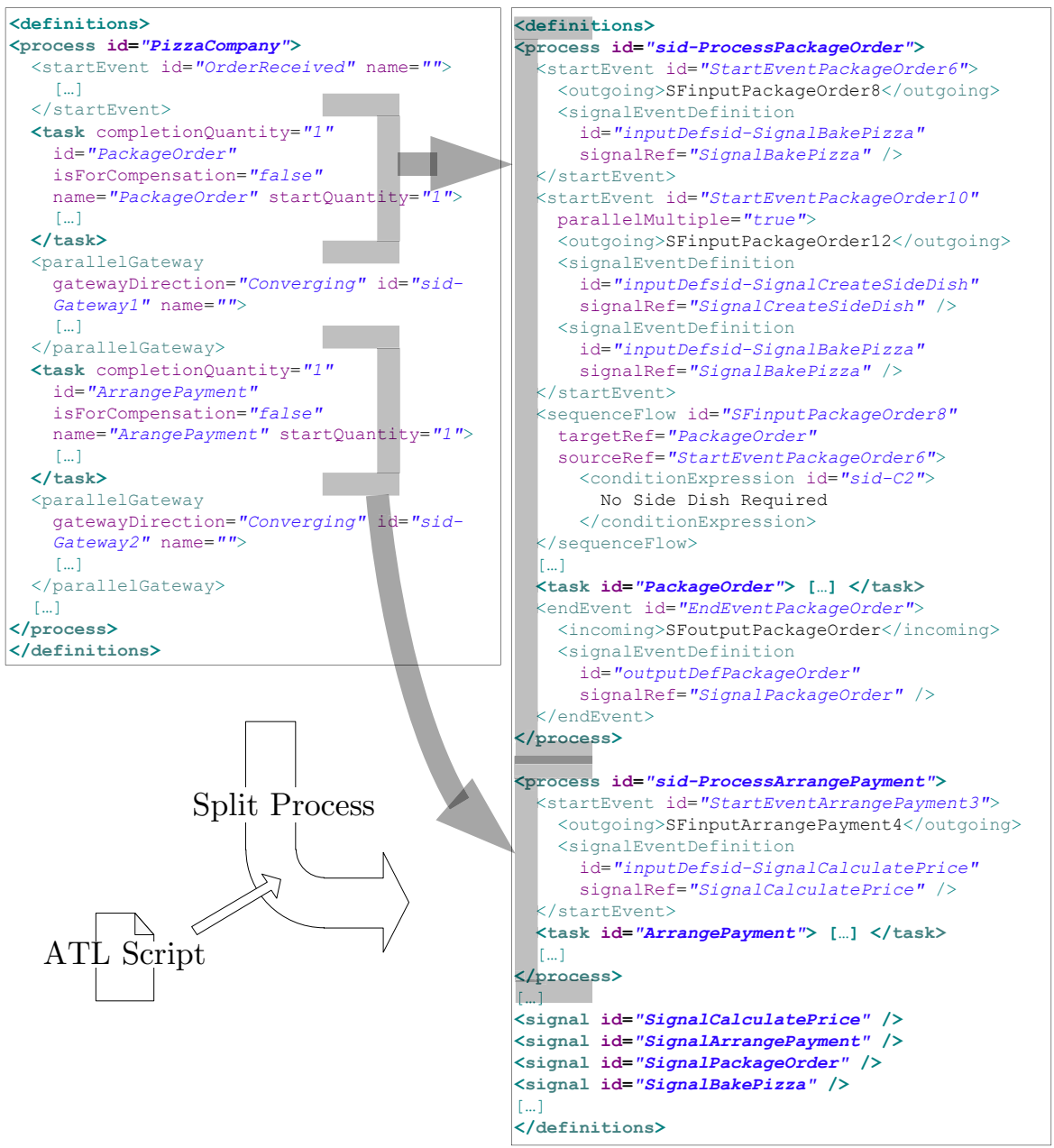

Fig. 7. Transformation of a global BPMN process to an event-based split BPMN process

event driven architecture 20]. A process engine should only be able to communicate with the event dispatchers in the event architecture.

The data payload (content) of an event message in the architecture should minimally consist of two things, one is the indication of the task it represents (e.g. the signal name found in the BPMN file, see Fig. 7), and another is a process instance id, indicating for which (global) process instance an action has been performed. The latter attribute is necessary to not loose the coupling between the process instance and the action performed. The payload of an event message can also be used to distribute any data related to the process execution (e.g. the event indicating the completion of CalculatePrice can incorporate the calculated price in the payload of the event message). 


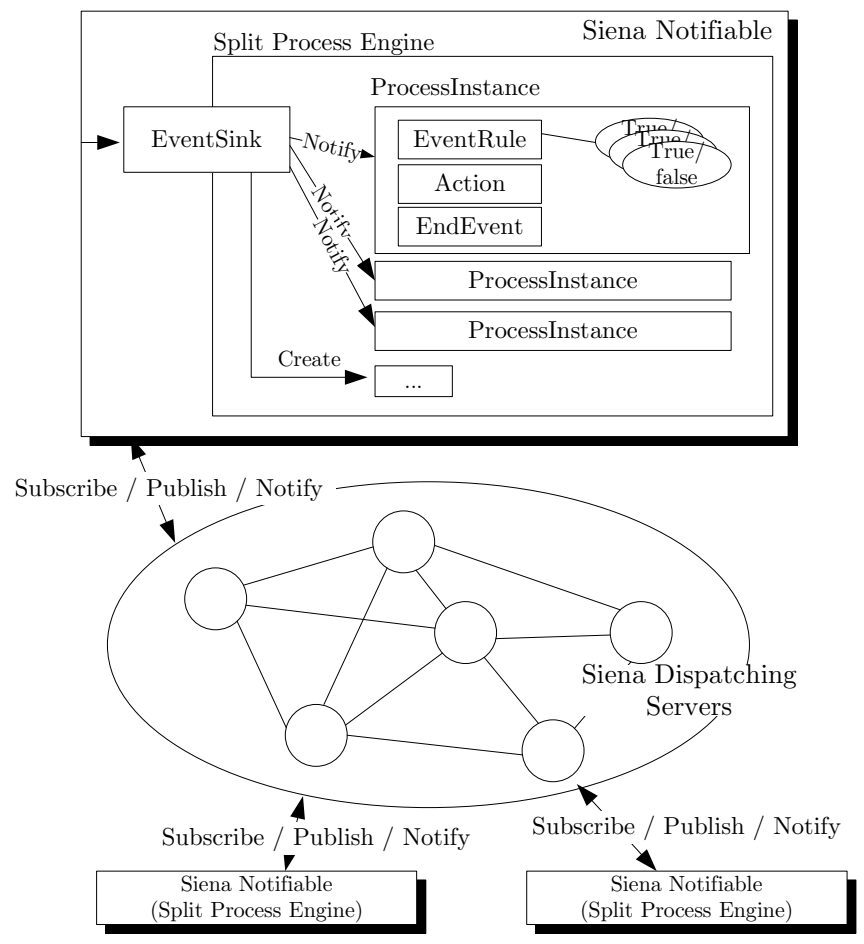

Fig. 8. Distributed Event-Based Orchestration Architecture

The working of a split process engine follows the BPMN2.0 execution semantics. Below, 3 steps are described which the process engine performs when an event notification arrives at its event $\operatorname{sink} 1$ (see Fig. 8).

1. The split process engine routes the event notification to the corresponding split process instance. This is done by matching the process instance id from the event notification (found in the data payload), with the process instance ids of its already running split process instances. If no match is found, a new split process instance is started (with id equal to the process instance id situated in the event notification payload) and the notification is routed to this new split process instance.

2. In the split process instance, the event notification is matched with the correct start event definition in its split process description (see e.g. Fig. 6). The corresponding event definition will be enabled (it holds a token).

3. For every enabled start event in the process flow (i.e. a conjunction in the starting-rule evaluates to true: every start event definition in the parallel multiple start event is enabled), the rest of the split process flow is interpreted and executed. Because we adopt a really fine grained distribution of the

${ }^{1}$ Note that we also started the formalization of the described publish/subscribe eventbased process execution [21, which we however omit here due to space limitations. 
global process flow, executing the distributed process flow usually means executing only one task (e.g. invoking the PriceCalculator service). When the process flow reaches its end event a notification is published by the process engine to signal the end of this split process instance (i.e. the completion of the executed task). Note that the split process instance isn't deleted from the system when it reaches its end event. It is possible that there are still tokens available in some start event definitions of the process instance and any additional (future) event notifications can again trigger the start of the same split process instance (this is true for non-safe process models, see Fig. 4).

The published end event is routed through the event architecture, and picked up by other interested split process engines, which handle this event again with the steps described above. Eventually, the combined execution of all these split process engines have achieved the global execution of the entire, designed process flow.

\section{Applicability}

In this section we will focus on the major added value of our decentralized eventbased orchestration, which are flexibility and adaptability. For tests on robustness and availability of distributed event based architectures (solving the single point of failure and performance bottleneck) we refer to extensive research done in the field of event based communication [1117], as well as to other research about decentralizing the process flow 3422 . The feasibility of our approach is demonstrated by our implementation of the transformation algorithm and our prototype execution architecture (see Sect. 4 and 5 ).

\subsection{Adaptability and Change Management}

The unawareness of interaction partners in an event-based communication creates a highly flexible infrastructure where components can enter and leave the architecture freely, without modifications to other components. Of course, when starting from a global process description, there is always a certain degree of dependency between the different split processes. It is designed by a process modeler that the task Arrange Payment should happen after the completion of task Calculate Price. Even with decentralized event communication, which creates a decoupling between these two tasks, the sequence dependency drawn by the process modeler still remains. Nonetheless, event communication adds some flexibility to process execution and adaptability. One advantage is a lesser change impact when re-deploying a redesigned process flow and another is the ability to autonomously change starting rules of a single split process instance.

Changing and re-deploying the global process flow. Due to the high degree of decoupling between the different split components (on execution level), respecifying and redeploying a previously deployed process model will have lesser 
Table 1. Change impact when changing the global process flow

\begin{tabular}{|c|c|c|}
\hline Change Pattern & Event Orchestration & Request Orchestration \\
\hline AP1-Serial Insert & 2 & 2 \\
\hline AP1-Parallel Insert & 2 & 3 \\
\hline AP1-Conditional Insert & 2 & 3 \\
\hline AP2-Delete Process Fragment & 2 & 3 \\
\hline AP3-Serial Move & 3 & 3 \\
\hline AP3-Parallel Move & 3 & 4 \\
\hline AP3-Conditional Move & 3 & 4 \\
\hline AP4-Replace Process Fragment & 2 & 2 \\
\hline AP5-Swap Process Fragment & 3 & 3 \\
\hline AP8-Embed Process Frag. in Loop & 2 & 2 \\
\hline AP9-Parallelize Process Frag. & $n+1$ & $\mathrm{n}+2$ \\
\hline $\begin{array}{|ll|}\text { AP10-Embed Process } & \text { Frag. in } \\
\text { Conditional Branch } & \\
\end{array}$ & 2 & 2 \\
\hline \begin{tabular}{|l|l|l|} 
AP11-Add Control Dependency \\
\end{tabular} & 1 & 2 \\
\hline \begin{tabular}{|l} 
AP12-Remove Control Dep. \\
\end{tabular} & 1 & 2 \\
\hline \begin{tabular}{|l} 
AP13-Update Condition \\
\end{tabular} & 1 & 1 \\
\hline AP14-Copy Process Fragment & 2 & 3 \\
\hline Total Components to Change & 32 & 4 \\
\hline
\end{tabular}

impact on the already running components than when using a request style of distributed orchestration. Table 1 counts the change impact according to the process change patterns introduced by Weber et al. [23. We compared the change impact of using an event-based communication style with the change impact of using a request based distributed orchestration [4] (Fig. 25 and b). To count the change impact, we counted, for a specific change pattern, the number of split components that need to be changed, where we assume that each component has a similar weight. For example, inserting a new task between the sequential tasks Calculate Price and Arrange Payment (change patten AP1-Serial Insert), has a change impact of 2 for event orchestration: the new inserted component and the next component in the sequence (the starting rule of Arrange Payment needs to change). When using a request-style of communication, also 2 components need to change, the new inserted component and the component preceding the new component in the flow (Calculate Price needs to send a request to the new component). From table 1 it can be seen that in 9 out of 16 cases, changing the process flow with event-based execution has lesser impact on the already running infrastructure. This is a substantial benefit, because change can be costly, certainly if the components are highly distributed and not readily available for change (e.g. other people are responsible).

With proper tool support and process instance management [6], only a limited amount of components need to be redeployed and the rest can be left untouched (and running) in the architecture.

Autonomously changing a split process at runtime. Another advantage of the unawareness of interaction partners on process execution level is the autonomy of each split process. The logic on when the split process needs to start 
is embedded in the split process itself. The split process has access to its own starting rules, independent of any other process engine in the global process infrastructure (unlike request based communication). This means that the entity (e.g. a person) responsible for the split process can change the starting rule of that split process independent of others. In our example, the pizza delivery boy can be responsible for his own split process holding the task Deliver Pizza (he has the split process running on a process engine on his PDA). Instead of waiting each time for the Arrange Payment AND Package Order tasks to complete, he can decide, for certain instances, to not wait for the Arrange Payment task and deliver the pizza nonetheless (e.g. the pizza is getting cold). Because each split process contains its own staring logic, the change is local and can be done without interfering with other split processes.

The split process engine could offer an interface to its manager, which enables creating and changing starting rules on the fly for specific process instances.

\section{Related Work}

In the domain of PAIS, the problem of centralized process execution is recognized by many researchers 5222243 . They all identify that, even though the actual service components are made reusable, distributed and loosely coupled through technologies like SOAP, WSDL and UDDI, the workflow- or process execution is still performed on a single central entity. Nanda et al. 24] use program dependency graphs, a tool borrowed from compiler optimization, to split up the process flow. Their goal is to reduce the network traffic involved. For the same reasons, Fdhila et al. 22] decentralize the process flow using dependency tables and Muth et al. 5] perform decentralization using state and activity charts. The eventual result is however always the same, a set of distributed control flows, where communication between the flows happens request-based. These solutions thus solve the technical issues of central orchestration (single point of failure and performance bottleneck), but still leave a tight coupled architecture, which affects robustness and adaptability.

The features of event communication are well researched in computer science 11. Event architectures have become a standard approach to create a loosely coupled and robust communication architecture. To reap the benefits of event communication, we leverage its advantages to distributed process execution. Notice that the combination of event driven architecture and service oriented architecture is a well known topic of research [25]. The focus of this research (EDA and SOA) is however on the invocation of services (open arrowhead arrows in Fig. 2), not on the decentralization of the process flow (full arrowhead arrows in Fig. 2).

In the domain of ubiquitous [14] and agent based [26] computing, the focus is also on event communication. This focus is complementary with our approach. Events generated by ubiquitous entities (e.g. RFID sensors) or agents can be incorporated in our infrastructure, so that split process engines react to these published events directly.

The flexibility and adaptability features we advocate in this paper are in complement with the research done on process adaptability 6]. For example, 
because of the decoupling features of event orchestration and the autonomy of split processes, the plug and play techniques of ADEPT [27] can easily be included in the architecture.

\section{Conclusion and Future Work}

We proposed a method that solves the issues of centralized process execution (single point of failure and performance degradation) and adds a layer of flexibility to the eventual process execution. We showed a non-intrusive transformation algorithm, that transforms a process model to smaller, event-based processes. This transformation happens at deployment time (in $O\left(n^{2}\right)$ time), without involvement of the process modeler. To illustrate the feasibility of the approach, we implemented the algorithm in the Atlas Transformation Language for process models defined with BPMN2.0 and developed a prototype execution architecture for the distributed event-based processes that are the result of the ATLtransformation. Each split process is run on a dedicated process engine, which differ both logically and physically from each other, and where communication between the engines is done with a publish/subscribe event-architecture. The advantage of this approach is that the decoupling features of event-based communication are adding flexibility to the process execution: there is a lesser impact on the running infrastructure when re-specifying and redeploying a process model, and the starting rules of any distributed process flow can be changed autonomic.

Future research involves working up the adaptability of distributed event-based execution and developing proper tool support to change the starting rules of split processes at run-time, as well as including split process instance management when changing the global process flow (which instances should be left running in the old configuration, which instances can change? [6]). We also intend to widen the scope of the transformable process elements to allow more specific process constructs (e.g. transactions) to be executed in this loosely coupled setting.

\section{References}

1. Dumas, M., Van Der Aalst, W., Ter Hofstede, A.: Process-aware information systems. Wiley Interscience, Hoboken (2005)

2. Coalition, W.M.: The workflow reference model. WfMC Documents (1995)

3. Schuler, C., Weber, R., Schuldt, H., Schek, H.J.: Scalable peer-to-peer process management-the osiris approach. In: IEEE Int. Conf. on Web Services (2004)

4. Chafle, G., Chandra, S., Mann, V., Nanda, M.: Decentralized orchestration of composite web services. In: Proceedings of the 13th International World Wide Web Conference on Alternate Track Papers \& Posters, pp. 134-143 (2004)

5. Muth, P., Wodtke, D., Weissenfels, J., Dittrich, A., Weikum, G.: From centralized workflow specification to distributed workflow execution. Journal of Intelligent Information Systems 10(2), 159-184 (1998)

6. van der Aalst, W., Basten, T., Verbeek, H., Verkoulen, P., Voorhoeve, M.: Adaptive workflow: on the interplay between flexibility and support. In: Enterprise Information Systems, pp. 63-70. Kluwer Academic Publishers, Norwell (2000) 
7. Recker, J.: Opportunities and constraints: the current struggle with BPMN. Business Process Management Journal 16(1), 181-201 (2010)

8. Object Management Group: Bpmn 2.0, beta 2 (June 2010), http://www.omg.org/cgi-bin/doc?dtc/10-06-04

9. Monsieur, G.: Pattern-based coordination in process-based service compositions, phd thesis. Phd Thesis, KULeuven (2010), https://lirias.kuleuven.be/ bitstream/123456789/284037/1/phd_geert_monsieur.pdf

10. Hens, P., Snoeck, M., De Backer, M., Poels, G.: Decentralized Event-Based Orchestration. In: Inter. Work. on Event-Driven Business Process Management. (2010)

11. Mühl, G., Fiege, L., Pietzuch, P.: Distributed Event-Based Systems. SpringerVerlag New York, Inc., Secaucus (2006)

12. Eugster, P., Felber, P., Guerraoui, R., Kermarrec, A.: The many faces of publish/subscribe. ACM Computing Surveys (CSUR) 35(2), 131 (2003)

13. Hallerbach, A., Bauer, T., Reichert, M.: Configuration and management of process variants. In: Handbook on Business Process Management, pp. 237-255 (2010)

14. Kong, J., Jung, J.Y., Park, J.: Event-driven service coordination for business process integration in ubiquitous enterprises. Computers \& Industrial Engineering 57(1), 14-26 (2009); Collaborative e-Work Networks in Industrial Engineering

15. Jouault, F., Allilaire, F., Bézivin, J., Kurtev, I., Valduriez, P.: ATL: a QVT-like transformation language. In: The 21st ACM SIGPLAN Symposium (2006)

16. Eclipse Project: Bpmn 2.0 ecore model, http://www.eclipse.org/projects/ project_summary.php?projectid=modeling.mdt.bpmn2

17. Carzaniga, A., Rosenblum, D.S., Wolf, A.: Design and evaluation of a wide-area event notification service. ACM Trans. Comput. Syst. 19(3), 332-383 (2001)

18. Niblett, P., Graham, S.: Events and service-oriented architecture: the OASIS web services notification specifications. IBM Systems Journal 44(4), 869-886 (2005)

19. Geppert, A., Tombros, D.: Event-based distributed workflow execution with EVE. In: Proc. of the IFIP Int. Conf. on Distributed Systems Platforms and Open Distributed Processing, pp. 427-442 (1998)

20. Juric, M.: WSDL and BPEL extensions for Event Driven Architecture. In: Information and Software Technology (2010)

21. Hens, P., Snoeck, M., De Backer, M., Poels, G.: A Petri Net Formalization of a Publish-Subscribe Process System. In: Submitted for the 5th ACM International Conference on Distributed Event-Based Systems (DEBS) (2011)

22. Fdhila, W., Yildiz, U., Godart, C.: A flexible approach for automatic process decentralization using dependency tables. In: ICWS 2009: Proceedings of the 2009 IEEE International Conference on Web Services, pp. 847-855. IEEE Computer Society, Washington, DC, USA (2009)

23. Weber, B., Reichert, M., Rinderle-Ma, S.: Change patterns and change support features-enhancing flexibility in process-aware information systems. Data \& Knowledge Engineering 66(3), 438-466 (2008)

24. Nanda, M., Chandra, S., Sarkar, V.: Decentralizing execution of composite web services. ACM SIGPLAN Notices 39(10), 170-187 (2004)

25. Michelson, B.: Event-driven architecture overview. OMG report (2006)

26. Wooldridge, M.: Agent-based software engineering. IEE Proceedings Software Engineering 144(1), 26-37 (1997)

27. Dadam, P., Reichert, M., Rinderle, S., Jurisch, M., Acker, H., Goser, K., Kreher, U., Lauer, M.: Towards truly flexible and adaptive process-aware information systems. In: Information Systems and e-Business Technologies, pp. 72-83 (2008) 